\title{
La tabla de Planeación Estratégica-Musical. Una herramienta para fortalecer el aprendizaje significativo-musical
}

\author{
Anderson Rodrigues da Silva
}

\section{Resumen}

El presente escrito tiene como objeto la propuesta de una herramienta gráfica para la optimización consciente y programada del aprendizaje musical: se trata de la tabla de Planeación Estratégica-Musical (tabla PEM). Con base en la premisa de que la acción de planear de forma estratégica potencializa el alcance de metas - tanto de manera individual como colectiva- de corto, medio y largo plazo, se hipotetiza que el uso de la tabla PEM puede optimizar la praxis musical debido a que su empleo permite a los músicos tener una mayor claridad en sus objetivos, proceso y conclusiones de sus propias prácticas y/o performances musicales. De esta forma, la tabla PEM promueve el enlace de los conocimientos previos con los hallazgos resultantes de una práctica coordinada con la cavilación en tres fases distintas, favoreciendo, así, un aprendizaje significativo-musical.

Palabras clave: música, planeación estratégica, optimización, aprendizaje significativo.

\section{Introducción}

Planear es una actividad inherentemente humana que conlleva a la organización de algunos objetivos para el alcance de un determinado fin. Desde la antigüedad -alrededor de 50,000 años atrás- se estima que los seres humanos ya planeaban sus acciones (Balter, 2015; Khan Academy, 2017). Por ello, esa actividad reflexiva que se ha desarrollado y evolucionado a lo largo de los siglos, permite que la toma de decisiones sea más acertada y coherente acorde al contexto en cuestión con el afán de obtener los resultados deseados.

La acción planeativa permite, entre otros aspectos, la identificación de puntos endebles en un determinado procedimiento, es decir, áreas que necesitan ajustes para que se alcance un fin específico. El estudio de la planeación, conforme aseveran Niño y Santamaría (2017), se fundamen- ta en los "análisis de la comprensión, del cómo el ser humano se relaciona con los fines para alcanzar los objetivos [...]" (p. 45), promueve una búsqueda sistémica, por lo que se puede hacer para el logro de metas individuales o colectivas. En tal sentido, Ferreira, Sykes y Batey (2009) señalan que la acción de planear puede incidir positivamente sobre un determinado fin que se pretenda alcanzar desde un ámbito conceptual y/o práctico.

Por otra parte, la literatura presenta una cierta variedad de tipos de planeación que difieren según el área donde se apliquen. Por ejemplo, Niño y Santamaría (2017) explican que, en efecto, hay diferentes modelos de planeación que son elaborados y aplicados por personas expertas en los más diversos sectores de las ciencias. En esta línea de ideas, Kunsch (2003) asevera que las planeaciones varían acorde a su aplicabilidad y que pueden ser complementarias, coexistentes e interdependientes 
en una organización. Por lo tanto, una planeación siempre va a adecuarse a un determinado propósito dentro de un ambiente específico, independientemente del área donde se emplee.

Con base en lo anteriormente expuesto, es factible asumir que el ejercicio de planear una determinada actividad en una arena musical -como en cualquier otra esfera del saber humano- puede favorecer el entendimiento acerca de esa práctica per se $\mathrm{y}$, por consiguiente, permitir una praxis musical más eficiente. En este mismo tenor, planear una actividad como un ensayo o un performance musical puede incrementar la calidad del quehacer en cuestión. Alves (2011) explica que esa acción previa al trabajo musical es de cabal importancia para que se puedan alcanzar los objetivos propuestos. Por lo tanto, fomentar esa práctica en el alumnado de música es fundamental para la optimización del aprendizaje de los procedimientos típicos del trabajo musical.

\section{La Planeación Estratégica}

La Planeación Estratégica (PE) es un término que comúnmente está relacionado con alguna institución. Chiavenato y Sapiro (2017) apuntan que la PE es la acción de planear generalmente llevada a cabo por una empresa. En ese marco, se puede pensar que la PE es una especie de herramienta de gestión $\mathrm{y}$, como tal, es una actividad deliberada de formulación e instauración de metas (Roncancio, 2018).

Desde una perspectiva educativa, la PE juega un papel de perentoria relevancia, puesto que ésta coadyuva a que los actores educativos alcancen las metas relacionadas con los procesos de enseñanza y aprendizaje de una manera más eficiente. Autores como Díaz-Barriga et al. (1990), Peralta (2016) y Aguilar (2018), entre otros, señalan la importancia de llevar a cabo una planeación para que el logro educativo ocurra de forma óptima en el ámbito educacional. A partir de este punto de vista, es coherente plantear que las y los estudiantes -independientemente del área de conocimiento-aprendan a identificar y organizar sus metas mediante una PE, de una manera que sepan qué hacer, cómo hacer y evaluar los resultados obtenidos.

Ya en la arena educativa-musical, sin embargo, aparentemente no hay todavía un consenso
《La interpretación musical es una acción

que requiere una consciencia previa al acto mismo del performance $\gg$

claro sobre la aplicabilidad de la PE en el quehacer musical porque trata de algo dúctil que se adecua siempre a un determinado contexto. No obstante, Barajas (2016) puntúa que la PE puede ejercer un sustancial papel para el éxito de un grupo musical. Asimismo, Rodrigues (2020) explica que la interpretación musical es una acción que requiere una conciencia previa al acto mismo del performance y señala la importancia de "formentar la práctica musical desde una perspectiva concienzuda, partiendo de metas claras y con la debida atención en el proceso para el logro" (p. 12); esto es, llevar a cabo la actividad músico-interpretativa a partir de una planeación.

\section{La tabla de Planeación Estratégica- Musical (PEM)}

La tabla PEM es una herramienta gráfica elaborada por el autor de este texto para coadyuvar el proceso de construcción de un determinado discurso musical, de tal manera que la herramienta en cuestión pueda ser usada para el establecimiento de puntos a ser trabajados u optimizados en una sección de estudio individual, un ensayo o en un performance musical -tomándose en cuenta una "compresión performativa" (Phelan, 2016, p. 104)-. Con base en la premisa de que una planeación consiste en una actividad de identificación y formulación de objetivos a ser trabajados, si esta actividad es realizada de una forma estratégica y contempla un objeto musical como meta, la tabla PEM puede servir para concatenar la información necesaria -de una manera organizada visualmente- para el logro musical en cuestión, en razón de que el procedimiento de llevar a cabo una PE, ayuda a que los individuos implicados en tal proceso alcancen sus metas (Cassidy, 2016).

La tabla PEM está conformada por tres dimensiones a ser tomadas en cuenta, de manera secuencial, para su implementación: objetivos (qué fines se desean alcanzar), proceso (cómo se llevarán a cabo las acciones para el logro de los objetivos) y 
conclusiones (una vez concretada la interpretación musical, cuáles resultados se obtuvieron y cuáles puntos deben de ser mantenidos, así como aquellos que requieren ajustes). A continuación, se desglosan más a detalle las mencionadas dimensiones:

- ¿Qué quiero? (objetivos) Aquí la idea es contemplar los objetivos a ser alcanzados durante el ensayo o el performance, de una manera inteligible, puntual y sucinta, con el propósito de clarificar qué se pretende con el discurso musical, siempre acorde al contexto de una determinada obra. Al escribir las metas ideadas, se maximiza el foco en los objetivos fijados, lo que puede contribuir con la conservación de la atención explícita en la acción musical misma.

Al plantearse las metas, ellas deben ser preferencialmente cortas y factibles para que, de esta forma, su logro sea optimizado (Rodrigues, 2019; Tichelaar et al., 2016). Así, al establecerse los propósitos, se puede contemplar con más facilidad qué objetivos se aspiran alcanzar con la actividad musical en cuestión.

- ¿Cómo lo haré? (proceso) En esta dimensión se plantea cuál será el procedimiento a realizar para el logro de las metas preestablecidas: cómo se ejecutará el instrumento musical -también puede aplicarse al canto y a la dirección musical- con la mira en el logro de los objetivos. De igual manera que la dimensión anterior, se mantiene la indicación de que la redacción debe ser lo más precisa posible, con la clara intención de objetivar al máximo cómo se darán los pasos determinados para el logro musical.

Al instaurarse la forma con la cual se concretarán los objetivos, se promueve la reflexión sobre la práctica musical per se, lo que genera la metacognición sobre el acto interpretativo-musical. Entonces, se puede considerar idóneo tal planteamiento porque, sin lugar a duda, refuerza la atención explícita en la praxis musical en virtud de los propósitos determinados, lo que puede producir un resultado significativo (Rodrigues, 2017). De igual manera que en otras áreas del conocimiento, como señalan Bexelius, Carlberg y Löwing (2018), la descripción procedimental ayuda al alcance del plan diseñado y, por consiguiente, cavilar acerca de un procedimiento musical para un objetivo específico puede favorecer el alcance de la meta propuesta.

- ¿Qué logré? (conclusiones) Esta dimensión de la herramienta, para cumplir de forma idónea con uno de sus propósitos -que es revisar la práctica con una postura reflexiva y, al mismo tiempo, crítica-, debe de ser contemplada justo después de la acción musical una vez concretada. Otra función que se aplica aquí es formular nuevos parámetros a ser trabajados, tomando en cuenta la propia interpretación musical llevada a cabo, cumpliendo, así, con esta parte de una PE.

De ese modo, es posible cavilar sobre la experiencia musical con el enfoque en determinar si los objetivos planteados y el proceso establecido fueron adecuados para la actividad realizada en cuestión. Esta reflexión es de suma importancia para la toma de decisiones que mejora la actuación musical (Bowman y Lucia, 2016; Witzleben, 2010). Asimismo, al suscitar más conciencia sobre qué se está haciendo y cómo se está llevando a cabo la interpretación musical, estas acciones permiten una experiencia significativa con la obra musical, lo que es sumamente positivo en el campo de la educación musical. Dicho de otro modo, la tabla PEM aspira promover, en consonancia con Estrada (2015), no un aprendizaje académico (conceptual), sino un aprendizaje vivencial (total), basado en las acciones ideadas y en la experiencia concretada de facto.

Ahora bien, se sugiere que las dimensiones estén divididas por apartados, de modo que la información sea concisa y de fácil visualización, lo que permite una interpretación rápida a primera vista (O’Donnell y Hurley, 2019). En la Tabla 1 se puede vislumbrar un modelo de la tabla PEM.

《Al plantearse cómo se concretarán

los objetivos, se promueve la reflexión sobre

la práctica musical per se, lo que genera

la metacognición sobre el acto interpretativo-musical $\gg$ 
Tabla 1. Modelo de la tabla PEM

\section{¿Qué quiero?}

Aquí se plantean, de forma sucinta, los objetivos contemplados para el estudio y/o el performance musical.

Ejemplo:

- Reforzar el discurso musical entre los números 5 y 6 de la obra de Castérède:

- Atentar al ritmo.

- Pulir el fraseo.

\section{¿Cómo lo haré?}

En este apartado se puntualiza el proceso a ser llevado a cabo en función de los objetivos planteados.

Ejemplo:

- Solfear el pasaje en cuestión, simulando las posiciones y asegurando la exactitud rítmica.

- Tocar con el instrumento el pasaje, haciendo el uso del metrónomo.

- Interpretar el pasaje, ahora, sin el uso del metrónomo, con atención en las terminaciones de las frases.

\section{¿Qué logré?}

Se sugiere que este apartado sea completado después del evento musical en cuestión, para indicar los aspectos que pueden ser mejorados, así como aquellos a ser mantenidos.

\section{Ejemplo:}

- El flujo melódico ha sido bueno y, por lo tanto, se puede mantener la simulación.

- El ritmo, sin embargo, aún no está del todo exacto. Trabajar una vez más con el metrónomo para asegurar este parámetro.

- Las terminaciones de las frases están más claras, pero se puede explicitar un poco más en la siguiente ocasión.

Fuente: Tabla elaborada por el autor.

\section{Conclusiones}

La herramienta presentada en este artículo está volcada para la optimización del quehacer práctico-musical a través de una PE. En un contexto educativo musical, la tabla PEM está ideada para que su uso implique una cavilación constante por parte de las y los estudiantes en la construcción de sus discursos musicales y la presentación de ellos durante tres fases distintas (antes, durante y después), de tal manera que la práctica aunada con la reflexión se paute en sus conocimientos previos, lo que potencia el aprendizaje significativo de este proceso constructivo-musical mediante una acción metacognitiva. En conformidad con Rodrigues (2019), la premisa de la tabla PEM consiste en el pensamiento arriba-abajo, esto es, en una proyección de la interpretación musical antes de que ésta ocurra (arriba) para su efectiva concreción (abajo).

En esta línea de ideas, este ejercicio de formulación (Roncancio, 2018) proporciona una re-mirada al quehacer musical, la cual permite repensar cómo llevar a cabo una acción interpretativa-musical a partir de una planeación -redactada, cavilada y fundada en la práctica musical misma de una determinada obra o estudio musical-, que pasa 
por su concretización y culmina en las conclusiones oriundas de este proceso. Por ello, es importante mencionar que ese planteamiento de la labor musical, basado en la utilización de la tabla PEM, no pretende ser considerado como una fórmula absoluta o cerrada, sino una propuesta de cómo construir un discurso musical de una manera eficiente $\mathrm{y}$, a la vez, significativa.

Asimismo, esta herramienta no está pensada para ser aplicada en una sola forma: cada tabla PEM es única y es diseñada por el propio individuo, lo que explora la creatividad y la flexibilidad del pensamiento musical y, por consecuencia, rompe con el esquema de "seguimiento de prácticas existentes" (Bowman y Lucia, 2016, p. 247). Por lo tanto, el uso de este instrumento
«El modelo de planeación estratégica planteado por el autor no se enfoca en el qué se va a tocar, y sí en el cómo se edificará el discurso musical \)

de PE en el quehacer músico-interpretativo, en el ámbito de la educación musical, puede enriquecer la praxis performativa e incrementar, por consiguiente, la calidad de la interpretación musical de quienes lo utilicen, debido a que la tabla PEM toma en cuenta la idiosincrasia de los individuos que la empleen. En otras palabras, el modelo de PE planteado por el autor no se enfoca en el qué se va a tocar, sino en el cómo se edificará el discurso musical, optimizando, así, el aprendizaje musical.

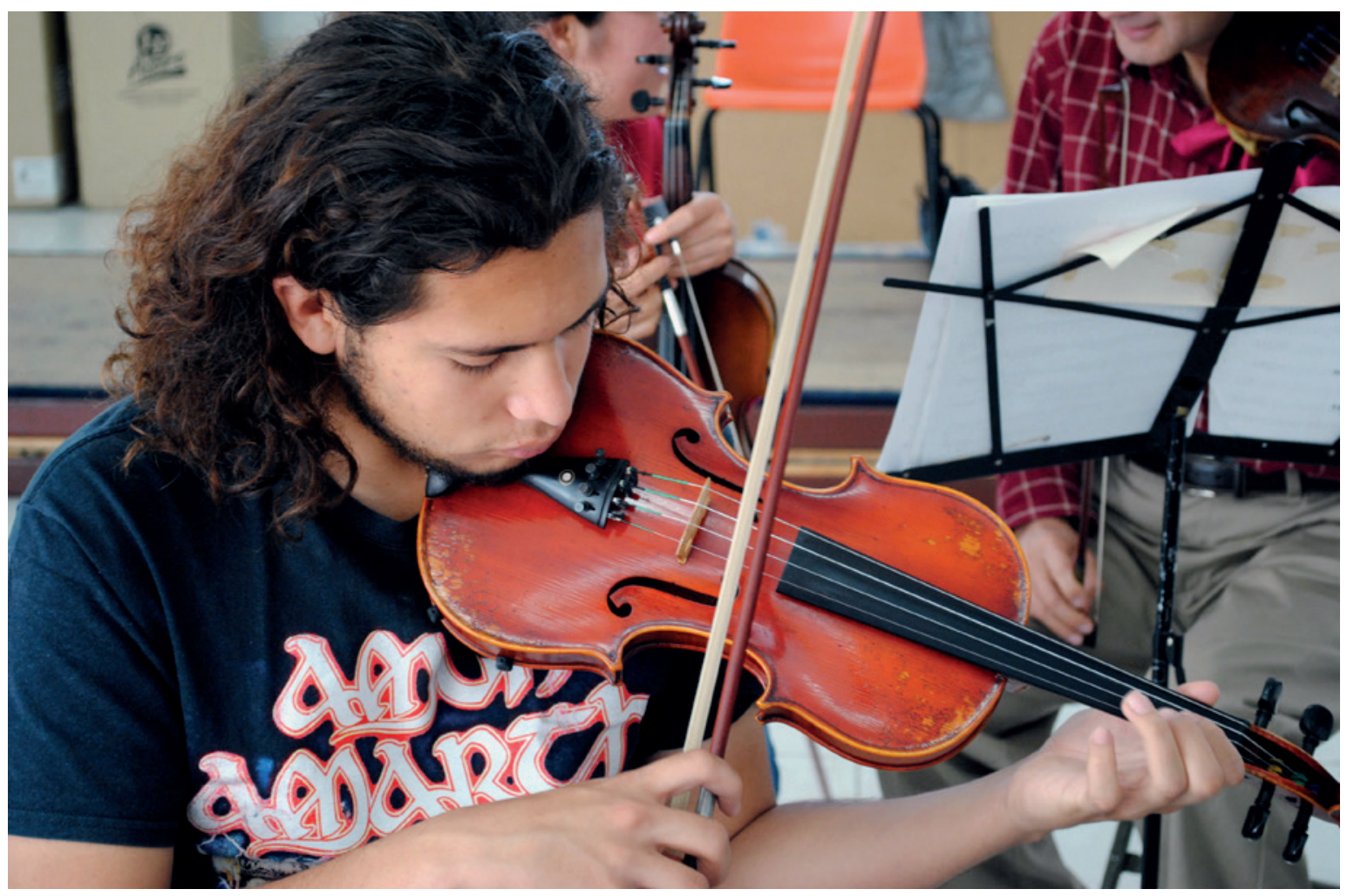

Fuentes de consulta

Aguilar, J. (2018). Planeación educativa y diseño curricular: un ejercicio de sistematización. Cathedra et Scientia International Journal, 4(1), 7-25. Recuperado de https://bit.ly/2MZSNCz.

Alves, L. (2011). O Ensaio-aula: uma proposta de metodologia de ensaio para banda de música. Revista Do Conservatório de Música, 4, 127-161.

Balter, M. (2015). Human language may have evolved to help our ancestors make tools. Science. Recuperado en https://bit.ly/3sY9FJa. 
Barajas, R. (2016). Iniciación y fundamentación de ensamble musical pop, con base en la planeación estratégica. Universidad Pedagógica Nacional.

Bexelius, A., Carlberg, E. y Löwing, K. (2018). Quality of goal setting in pediatric rehabilitation-A SMART approach. Child: Care, Health and Development, 44(6), 850-856. Recuperado de https:// doi.org/https://doi.org/10.1111/cch.12609.

Bowman, W. y Lucia, A. (2016). ¿Pero esto es Filosofía? Manual Oxford de Filosofía en Educación Musical, 242-247. Sb editorial.

Cassidy, A. (2016). A practical guide to information systems strategic planning. Aurbach Publications.

Chiavenato, I. y Sapiro, A. (2017). Planeación estratégica. Fundamentos y aplicaciones. Mc GrawHil Education.

Díaz-Barriga, F., Lule, F., Rojas, M. y Saad, S. (1990). Metodología de diseño curricular para educación superior. En Metodología de Diseño Curricular para la Educación Superior, 46-158. Trillas.

Estrada, M. (2015). Relaciones humanas (2a ed.). El Manual Moderno.

Ferreira, A., Sykes, O. y Batey, P. (2009). Planning Theory or Planning Theories? The hydra model and its implications for planning education. Journal for Education in the Built Environment, 4(2), 29-54. Recuperado de https:doi.org/10.11120/jebe.2009.04020029.

Khan Academy. (2017). Homo sapiens and early human migration. Khan Academy. Recuperado de https://bit.ly/3t4Ek7W.

Kunsch, M. (2003). Planejamento de relações públicas na comunicação integrada. Summus.

Niño, D. y Santamaría, Y. (2017). Una aproximación teórica a la planeación en las organizaciones. Revista Questionar, 5(1), 43-53. Recuperado de https://bit.ly/2N5iphA.

O’Donnell, L. y Hurley, M. (2019). How to create tables from data. Writing clear science. [Página web]. Recuperado de https://bit.ly/38rU5xE.

Peralta, C. (2016). Adecuación de la planeación didáctica como herramienta docente en un modelo universitario orientado al aprendizaje. REICE. Revista Iberoamericana Sobre Calidad, Eficacia y Cambio En Educación, 14(3), 109-130. Recuperado de https://doi.org/doi:10.15366/reice2016.14.3.006.

Phelan, H. (2016). Dándole voz a los "Imbas": llevando a la práctica una filosofía de la educación musical. En W. Bowman y L. Frega (Eds.), Manual Oxford de Filosofía en Educación Musical, 100109. Sb editorial.

Rodrigues, A. (2017). Educación emocional como apoyo a la educación musical. Revista Vinculando, 1-11. Recuperado de http://bit.ly/30p9hHw.

Rodrigues, A. (2019). El desarrollo de estrategias educativas basadas en la Inteligencia Emocional para mejorar la calidad músico-interpretativa de estudiantes adolescentes. Universidad Cuauhtémoc. Recuperado de https://bit.ly/3enczDx.

Rodrigues, A. (2020). El sistema EMA-EMAI: una forma heurística de apoyar la formación músico-interpretativa. Revista Arte, Imagen y Sonido, 1(1), 1-17. Recuperado de http://bit.ly/38o8Odd.

Roncancio, G. (2018). ¿Qué es la Planeacion Estratégica y para qué sirve? Pensemos. [Página web]. Recuperado de https:/gestion.pensemos.com/que-es-la-planeacion-estrategica-y-para-que-sirve.

Tichelaar, J., Den, S., Antonini, N., van Agtmael, M., de Vries, T. y Richir, M. (2016). A “SMART” way to determine treatment goals in pharmacotherapy education. British Journal of Clinical Pharmacology, 82, 280-284. Recuperado de https://doi.org/https://doi.org/10.1111/bcp.12919.

Witzleben, J. (2010). Performing in the shadows: learning and making music as ethnomusicological practice and theory. Yearbook for Traditional Music, 42, 135-166. Recuperado de https://bit.ly/3eB1lvl. 